\title{
Relative Incidence of Nasal Masses: A Tertiary Care Hospital Experience
}

\author{
Jaskaran Singh ${ }^{1}$, Bhanu Bhardwaj ${ }^{2}$, Tanya Singh ${ }^{3}$
}

\begin{abstract}
Introduction: Nasal masses are an intriguing arena for a rhinologist. With diagnostic advancement from anterior rhinoscopy to threedimensional endoscopic view at a blazing speed in rhinology, it has become easier to diagnose a nasal mass. Early detection is a key for better management. Incidence of an entity varies over time because of the ever-changing environmental scenario and availability of advanced diagnostics. Incidence of nasal masses is still of importance because the pathophysiology of the nasal masses is still under research. This study will bring into notice of a rhinologist the relative incidence of various nasal masses highlighting the areas of concern and hence bringing our focus to a better management.

Materials and methods: It is a prospective study with a sample size of 200. All the modern diagnostic facilities were used, including a computed tomography scan can and nasal endoscopy, to reach a presumptive diagnosis of various nasal masses, and histopathology was done to establish the final diagnosis.

Results: In the present study, $62 \%$ were males (124) while $38 \%$ were females (76). Majority of the patients were in age-group of $21-40$ years $(42.5 \%, n=85)$, followed by $40 \%(n=80)$ in the age-group $41-60$ years, and $14.5 \%(n=29)$ in 10 -20-year age-group. Around $6(3 \%)$ cases were in less than 10 years of age-group. Out of 200 cases, 160 cases were non-neoplastic masses. Out of 40 neoplastic masses, 24 were benign and 16 were malignant. The most common mass was nasal polyps (144 of 200).

Conclusion: Nasal polyps are still the most common nasal masses. Improvement in diagnostic modality mandates a more active research to understand their molecular biology for better management.

Keywords: Incidence, Nasal mass, Nasal obstruction, Study.

Clinical Rhinology An International Journal (2019): 10.5005/jp-journals-10013-1356
\end{abstract}

\section{INTRODUCTION}

Nasal masses and nasal obstruction are common complaints encountered in otorhinolaryngology clinics. There is a changing trend in the incidence of nasal masses. Diagnostic algorithm of nasal masses continue to evolve along with advances in rhinological techniques. Accurate diagnosis helps the surgeon to manage the disease early. ${ }^{1}$ Improved visualization of sinonasal cavities is substantially responsible for improved surgical management of nasal pathologies. ${ }^{2}$

The aim of the present study aims to evaluate the incidence of various nasal masses in a tertiary care hospital.

\section{Materials and Methods}

The present study entitled "Relative Incidence of Nasal Masses: A Tertiary Care Hospital Experience" was conducted in Department of ENT, Shri Guru Ram Das Medical College and Research Institute Amritsar, Punjab, India, from September 2015 to August 2016.

Source of data: All patients attending ENT OPD with nasal complaints who were willing to undergo diagnostic and therapeutic modalities.

Sample size: 200 .

\section{Inclusion Criteria}

All patients up to 70 years with mass in nose and nasopharynx having complaints of bleeding from nose, nasal obstruction, and nasal discharge.

\footnotetext{
1,2Department of ENT, Shri Guru Ram Das Institute of Medical Sciences and Research, Amritsar, Punjab, India

${ }^{3}$ Department of ENT, Career Institute of Medical Sciences and Hospital, Lucknow, Uttar Pradesh, India
}

Corresponding Author: Bhanu Bhardwaj, Department of ENT, Shri Guru Ram Das Institute of Medical Sciences and Research, Amritsar, Punjab, India, Phone: 9815201845, e-mail: entwithdrbhanu@gmail. com

How to cite this article: Singh J, Bhardwaj B, Singh T. Relative Incidence of Nasal Masses: A Tertiary Care Hospital Experience. Clin Rhinol An Int J 2019;12(1):16-20.

Source of support: Nil

Conflict of interest: None

\section{Exclusion Criteria}

Patients not giving consent to participate.

The selected cases were subjected to a detailed clinical examination after proper history. These patients underwent a diagnostic nasal endoscopy using standard three-pass technique, and findings were noted.

All the patients who had a nasal mass underwent a computed tomography (CT) scan plain plus contrast in axial, coronal, and sagittal views to access the exact extent of the disease. All the patients were subjected to endoscopic sinus surgery for biopsy or complete removal of the mass per the differential diagnosis on CT scan in accordance with management strategies used in standard

() The Author(s). 2019 Open Access This article is distributed under the terms of the Creative Commons Attribution 4.0 International License (https://creativecommons. org/licenses/by-nc/4.0/), which permits unrestricted use, distribution, and non-commercial reproduction in any medium, provided you give appropriate credit to the original author(s) and the source, provide a link to the Creative Commons license, and indicate if changes were made. The Creative Commons Public Domain Dedication waiver (http://creativecommons.org/publicdomain/zero/1.0/) applies to the data made available in this article, unless otherwise stated. 
practice. The specimen was preserved in formalin solution and sent for histopathological examination. The data so obtained were charted and results were analyzed.

\section{Results}

In the present study, 62\% (124) were males while 38\% (76) were females. Majority of the patients were in age-group 21-40 years $(42.5 \%, n=85)$, followed by $40 \%(n=80)$ in age-group $41-60$ years, and $14.5 \%(n=29)$ in the $10-20$-year age-group. Around $6(3 \%)$ cases were in less than 10 years of age-group. Out of 200 cases, 160 cases were non-neoplastic masses; 40 of 200 cases were neoplastic masses (Table 1). Out of 40 neoplastic masses, 24 were benign and 16 were malignant (Table 2 and Flowchart 1). The most common mass was nasal polyps ( 144 out of 200 ) (Table 1): $77.38 \%$ were ethmoidal polyps, while $23.16 \%$ were antrochoanal polyps and 8 patients were mucoceles (Tables 1 and 3), rhinoscleroma and rhinosporidiosis were seen in 2 and 6 cases, respectively (Tables 1 and 3).

Out of benign tumors, juvenile nasal angiofibroma was seen in 12 cases followed by inverted papilloma (8 cases) and osteoma (4 cases), respectively (Table 4 ).

Squamous cell carcinoma (SCC) was the most common malignant tumor in eight cases, while Ca nasopharynx was seen in six cases. Malignant melanoma was seen in 2 cases (Table 5).

Table 1: Relative incidence of various nasal masses

\begin{tabular}{lcc}
\hline Nasal mass & Number of cases & $\%$ \\
\hline Nasal polyps & 144 & 72 \\
Mucocele & 08 & 4 \\
Rhinosporidiosis & 06 & 3 \\
Rhinoscleroma & 02 & 1 \\
Angiofibroma & 12 & 6 \\
Inverted papilloma & 08 & 4 \\
Osteoma & 04 & 2 \\
Squamous cell carcinoma & 08 & 4 \\
Carcinoma nasopharynx & 06 & 3 \\
Malignant melanoma & 02 & 1 \\
\hline
\end{tabular}

Table 2: Relative incidence of the neoplastic and non-neoplastic masses

\begin{tabular}{lll}
\hline Nature & Number of cases & $\%$ \\
\hline Non-neoplastic & 160 & 80 \\
Neoplastic & 40 & 20 \\
Benign & 24 & 12 \\
Malignant & 16 & 8 \\
\hline
\end{tabular}

\section{Discussion}

The nasal masses in our study showed a male preponderance. Study by Zafar et al. also shows a male preponderance, although a study from Nigeria shows a female preponderance. ${ }^{3,4}$ Majority (80\%) of the cases in our study were non-neoplastic masses. Lathi et al. also found $71.4 \%$ of sinonasal masses to be non-neoplastic, and this is supported by Zafar et al. and Bakari et al. ${ }^{3-5}$ Bist et al. found the number of non-neoplastic lesions to be $60 \%$ compared to $4 \%$ neoplastic lesions in their study. In the neoplastic group, $19.8 \%$ of the patients were benign, while $23.76 \%$ were malignant. ${ }^{6}$ Khan et al. had $60 \%$ of their cases as non-neoplastic $(n=240)$. Out of this $40 \%$ cases, $23.33 \%$ were benign and $16.67 \%$ were malignant. ${ }^{7}$

Nasal polyps was the most common lesion observed in our study (Fig. 1). Of our 200 cases, 144 patients presented with nasal polyposis. A study by Bhattacharya et al. in population of north Bengal and Modh et al. also had similar results, with nasal polyps being the most common nasal masses. ${ }^{1,2}$ Bist et al. had incidence of $80.33 \%$ in their non-neoplastic group. ${ }^{6}$ Tandon et al. and Dasgupta et al. reported an incidence of nasal polyps to be $47 \%$ and $31.98 \%$

Table 3: Relative age-specific and gender-specific incidence of nonneoplastic masses (160 cases)

\begin{tabular}{clll}
\hline Type of nasal mass & No. of patients & Male & Female \\
\hline Nasal polyps & $144(90 \%)$ & 88 & 56 \\
$<10$ years & 6 & 4 & 2 \\
$10-20$ years & 16 & 10 & 6 \\
$21-40$ years & 72 & 44 & 28 \\
$41-60$ years & 50 & 30 & 20 \\
Mucocele & $08(5 \%)$ & 6 & 2 \\
$<10$ years & None & None & None \\
$10-20$ years & None & None & None \\
$21-40$ years & None & None & None \\
$41-60$ years & 8 & 6 & 2 \\
Rhinosporidiosis & $06(3.7 \%)$ & 4 & 2 \\
$<10$ years & None & None & None \\
$10-20$ years & None & None & None \\
$21-40$ years & 6 & 4 & 2 \\
$41-60$ years & None & None & None \\
Rhinoscleroma & $02(1.2 \%)$ & 2 & None \\
$<10$ years & None & None & None \\
$10-20$ years & None & None & None \\
$21-40$ years & 2 & 2 & None \\
$41-60$ years & None & None & None \\
\hline
\end{tabular}

Flowchart 1: Histopathological division of various nasal masses in our study

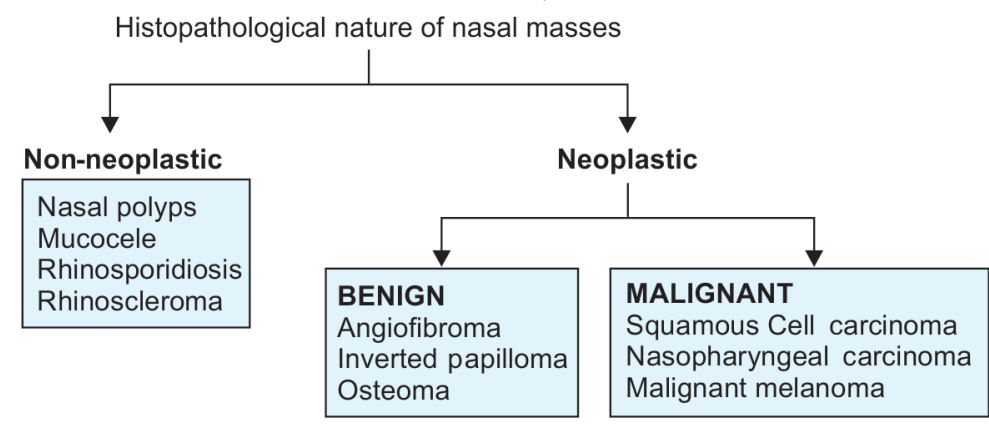


Table 4: Relative age-specific and gender-specific incidence of benign neoplastic masses ( 24 cases)

\begin{tabular}{clll}
\hline Type of nasal mass & No. of patients & Male & Female \\
\hline Angiofibroma & $12(50 \%)$ & 12 & None \\
$<10$ years & None & None & None \\
10-20 years & 12 & 12 & 12 \\
$21-40$ years & None & None & None \\
$41-60$ years & None & None & None \\
Inverted papilloma & $08(33.33 \%)$ & 6 & 2 \\
$<10$ years & None & None & None \\
$10-20$ years & None & None & None \\
$21-40$ years & 2 & 2 & None \\
$41-60$ years & 6 & 4 & 2 \\
Osteoma & $04(16.6 \%)$ & 2 & 2 \\
$<10$ years & None & None & None \\
$10-20$ years & 1 & None & 1 \\
$21-40$ years & 1 & 1 & None \\
$41-60$ years & 2 & 1 & 1 \\
\hline
\end{tabular}

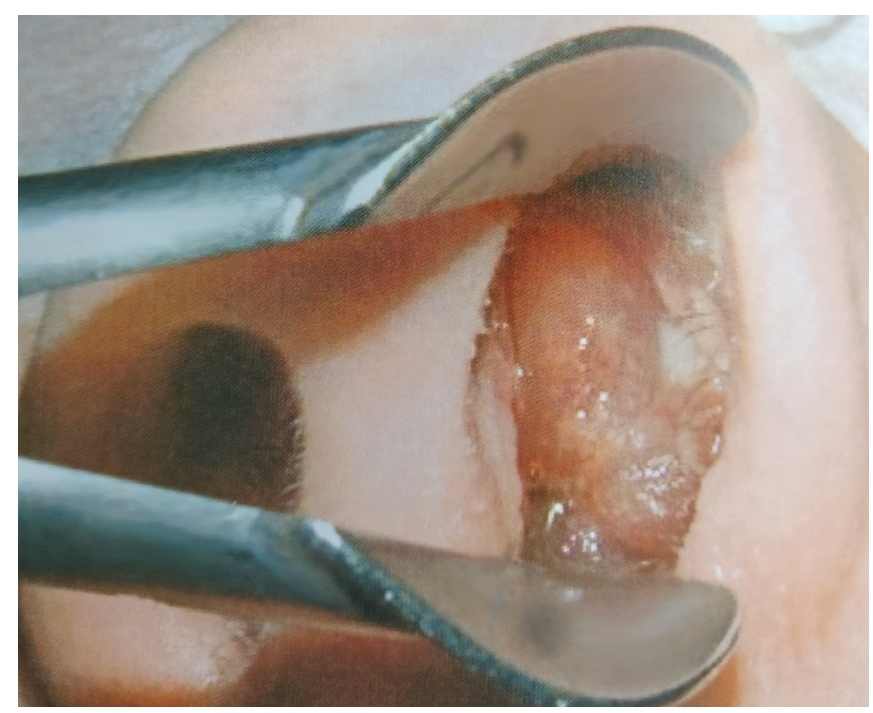

Fig. 1: Photograph showing nasal polyp on anterior rhinoscopy in one of our patients

of all cases. ${ }^{8,9}$ Khan et al. reported 120 of 240 cases to be nasal polyps. ${ }^{7}$ The main complaints of the patients were nasal blockage, nasal mass, rhinnorhea, and headache. In our study, ethmoidal polyps was more common than antrochoanal polyps. Drake Lee also found similar results. ${ }^{10}$ In the present study, there was a male preponderance. Zafar et al. also showed a male to female ratio of 1.7:1. ${ }^{3}$ Most of our patients with nasal polyps were in the second to fourth decade of life followed by those in fourth to sixth decade of life. Children were less affected. According to Bellanti et al., along with anatomic and physiologic changes of nose, nonspecific immune changes contribute more to allergic mechanisms as the age progresses. ${ }^{11}$ Polyps are known to have a clear association with asthma, aspirin sensitivity, and cystic fibrosis. Over past 10 years, research has revealed unique cytokine and cellular inflammatory profiles contributing to nasal polyposis. Approximately $80 \%$ of patients in Western countries are characterized by a robust Th2 response, eosinophilic infiltration, and decreased $\mathrm{T}$ regulatory
Table 5: Relative age-specific and gender-specific incidence of malignant neoplastic masses (16 cases)

\begin{tabular}{clll}
\hline Type of nasal mass & No. of patients & Male & Female \\
\hline Squamous cell carcinoma & $08(50 \%)$ & 5 & 3 \\
$<10$ years & None & None & None \\
$10-20$ years & None & None & None \\
$21-40$ years & None & None & None \\
$41-60$ years & 8 & 5 & 3 \\
Cancer nasopharynx & $06(37.5 \%)$ & 6 & None \\
$<10$ years & None & None & None \\
$10-20$ years & None & None & None \\
$21-40$ years & 2 & 2 & None \\
$41-60$ years & 4 & 4 & None \\
Malignant melanoma & $02(12.5 \%)$ & 2 & 2 \\
$<10$ years & None & None & None \\
$10-20$ years & None & None & None \\
$21-40$ years & None & None & None \\
$41-60$ years & 2 & None & 2 \\
\hline
\end{tabular}

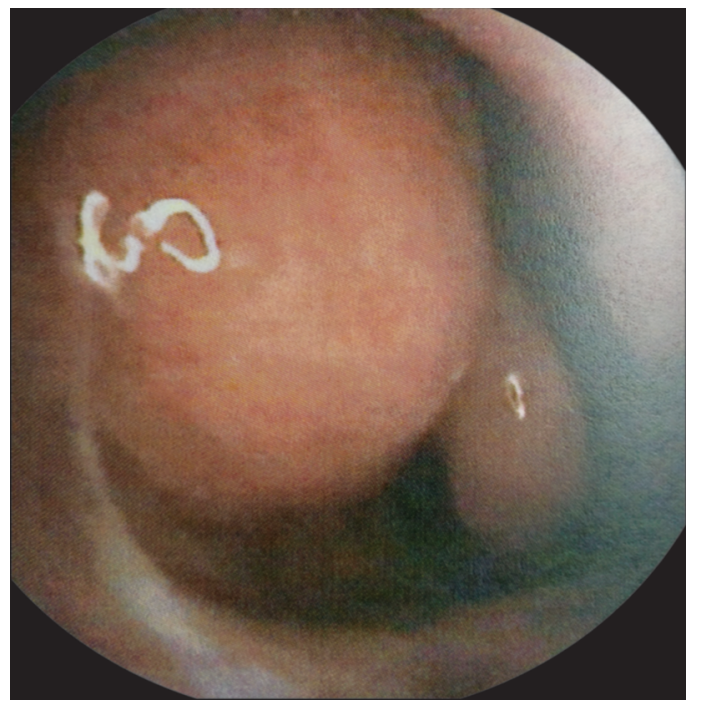

Fig. 2: Endoscopic picture showing frontoethmoidal mucocele pushing the middle turbinate towards septum

function. This is consistent with studies in Asian countries. Some studies indicate the role of fungi and bacteria in nasal polyps. Their cause however remains unknown, and it is possible that it is not same in all the patients. ${ }^{12-14}$

Mucoceles are dilated mucous containing sacs lined by squamous epithelium..$^{15}$ In the present study, there were eight mucoceles (Table 1 and Fig. 2) with common complaints of swelling of medial canthus of eye, increased watering, and proptosis. A study by Suri et al. reported proptosis (83\%) and diplopia (45\%) as most common complaints. ${ }^{16}$ In our study, six patients were males and two were females. There was history of trauma in four cases. The etiology of mucoceles is multifactorial which involves inflammation allergy trauma previous surgery. ${ }^{15} \mathrm{Clinical}$ presentation varies from asymptomatic to incapacitating headache or proptosis. Dermoid cysts, histiocystosis, and fungal and tubercular infection along with frontoorbital cholesterol granuloma must be considered in differential diagnosis. ${ }^{17}$ 


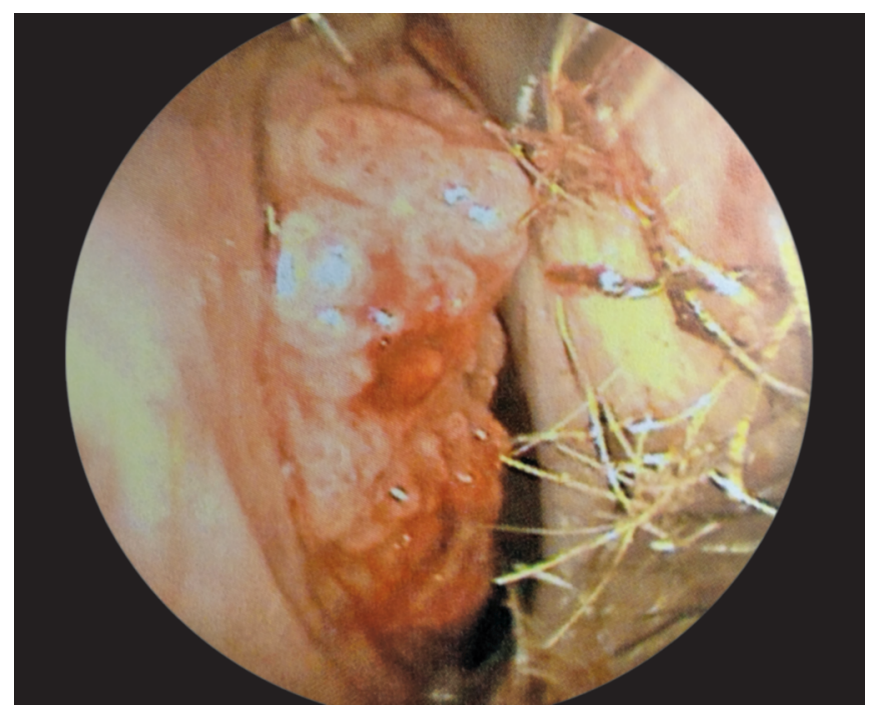

Fig. 3: Endoscopic picture showing rhinosporidiosis

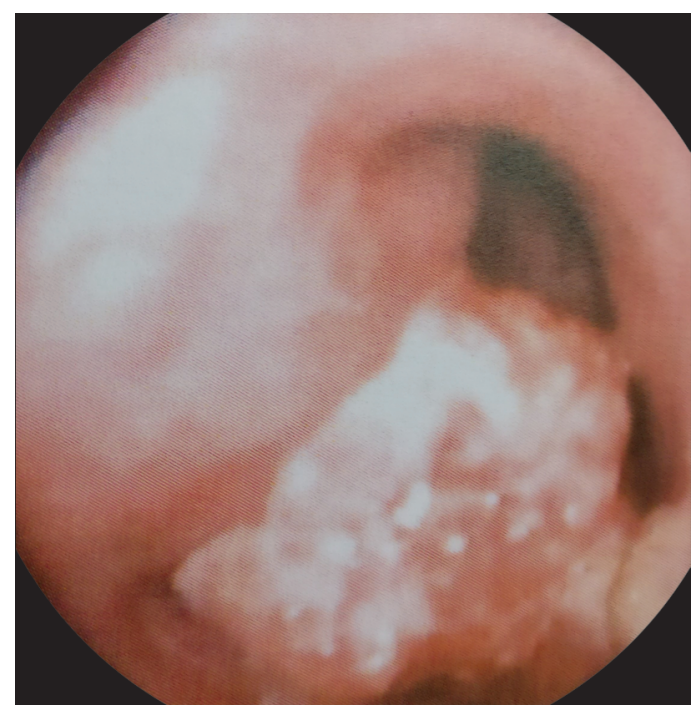

Fig. 5: Endoscopic picture showing inverted papilloma

Rhinoscleroma is endemic Egypt as well as sporadic worldwide including central America, Africa, and India. ${ }^{18}$ Rhinoscleroma was seen in 1\% (Table 1) of cases in our study and the age-group of the patients was 21-40 years. Most studies agree to this, as they also quote this disease usually presents in second to third decade of life. ${ }^{7-9}$ However, study by Lathi et al. reported a higher incidence of $10 \%$ which is not supported by studies by Zafar et al., Pradhanga et al., and Bakhari et al. ${ }^{3-5,19}$

The symptoms were nasal obstruction and foul-smelling discharge. ${ }^{20}$

Rhinosporidiosis was present in six cases, that is about $3 \%$ (Table 1 and Fig. 3). Lathi et al. found only two cases out of their 112 cases, while Pradhanga et al. found only one case during their 2 year study, whereas Bist et al. found no case in their cases of 110 patients. ${ }^{5,6,19}$

Out of neoplastic masses in our study, nasal angiofibroma was seen in 12 of 200 cases (Table 1 and Fig. 4). It was the most common benign tumor encountered in our study. (Table 4). Khan et al. also

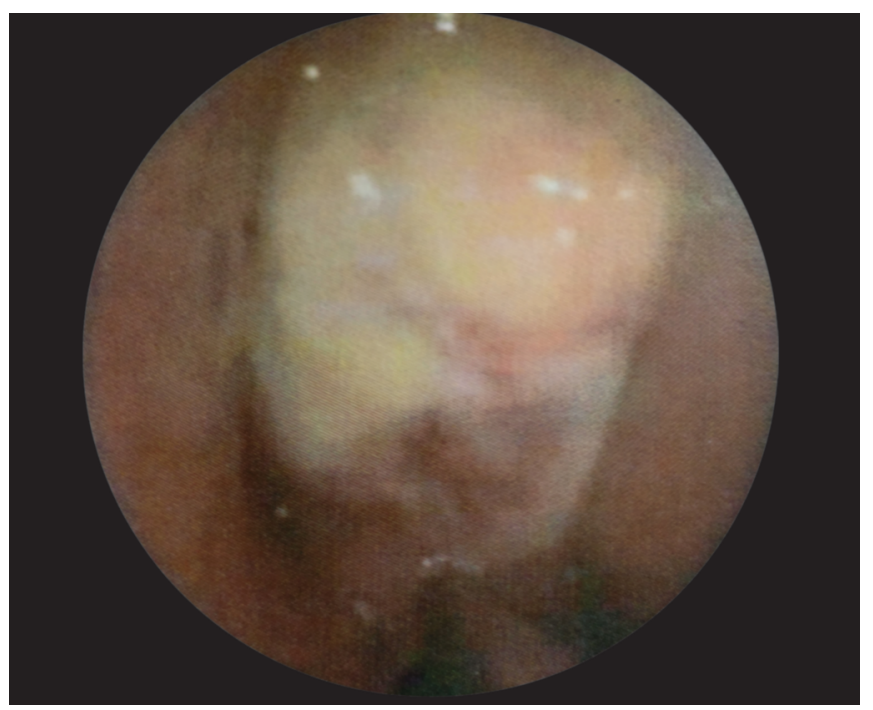

Fig. 4: Endoscopic picture of nasal angiofibroma after embolization

report $42.5 \%$ (24) cases out of their 56 cases of benign tumors to be angiofibromas in their study of 96 cases. ${ }^{7}$ Nasal angiofibroma was the most common benign tumor seen in $35 \%$ of cases in one study, while another study quotes only one case. ${ }^{5,6}$ Pradhanga et al. reported nine cases, while angiofibromas were reported to be common in Europe forming $0.5 \%$ of all head and neck cases. ${ }^{5,19}$

Inverted papilloma was seen in eight cases (Table 1 and Fig. 5). The reported incidence of papilloma in the literature varies from 0.4 to $4.7 \% .{ }^{21}$ In our study, the male to female ratio was $3: 1$. This male preponderance is virtually in all series according to Lawson et al. and Vrabec et al. ${ }^{22,23}$ The peak age incidence was seen in fifth decade. This was in accordance with other studies. ${ }^{24,25}$ The main presentation is mass in nasal cavity with blood-stained discharge. They are commonly confused clinically with a fibrosed antrochoanal polyp and affecting the surgical management and leading to recurrence. Suspicion of inverted papilloma should arise intraoperatively if mass arising from lateral nasal wall is firm, more extensive than expected, bleeds more, and cannot be removed in toto with a stalk. ${ }^{26}$ They were the second most common benign tumors in the present study (33.3\%) (Table 4). This was in accordance with study by Lathi et al. in which the lesion formed $36.4 \%$ of all benign tumors but was higher than that reported by Bakri et al. ${ }^{4,5}$ Khan et al. also reported inverted papilloma to be the second most common benign tumor (26.5\%). ${ }^{7}$

Osteoma was seen in four cases all in the age group of 20-40 years with equal preponderance in both sexes (Table 1). Lathi et al. did not report a single case in their study. Bist et al. also did not encounter any case. ${ }^{5,6}$

Out of neoplastic lesions, SCC was the most common. (Table 5) eight (50\%) of 16 cases were seen. They constituted $7 \%$ of all lesions. The most common age-group was 40-60 years with male to female ratio of 2:1. Khan et al. also observed similar results. ${ }^{7}$ Lathi et al. showed SCC carcinoma forming $11.6 \%$ of all lesions and $40.6 \%$ of neoplastic masses in their study. Svane Kudsoon et al. have also reported similar results in their study in Denmark. Pradhananga et al. report $6.3 \%$ of their sinonasal masses to be malignant., 5

There were six cases of nasopharyngeal cancer which constituted $37.5 \%$ of all malignant lesions (Table 1 and Fig. 6). Majority were seen above fourth decade of life. Khan reported an 


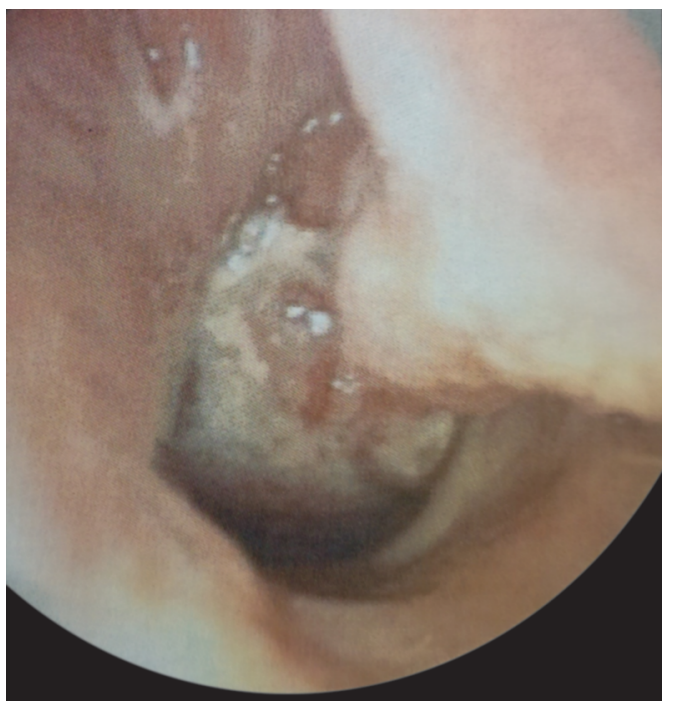

Fig. 6: Endoscopic picture showing proliferative growth in nasopharynx which on histopathology was diagnosed as nasopharyngeal carcinoma

incidence of $25 \%$ out of his malignant cases. ${ }^{7}$ Two of 16 neoplastic cases were malignant melanoma with total female preponderance in our study.

\section{Conclusion}

Nasal masses are still a topic of major interest. Since nasal polyps are still the most common nasal masses, we conclude that we need more research in the pathogenesis of nasal polyps for better management. With changing environmental scenarios there is a rise in nasal malignancies for which an effective management at molecular level is still under research. Improvement in diagnostics has led to better diagnosis, but a significant shift in management still requires a contributory effort from all rhinologists working at various levels.

\section{References}

1. Modh SK, Delwadia KN, Gonsai RN. Histopathological spectrum of sinonasal masses-a study of 162 cases. IJCRR 2013;5(3):83-91.

2. Bhattacharya J, Goswami BK, Banerjee A, et al. A clinicopathological study of masses arising from sinonasal tract and nasopharynx in north Bengal population with special reference to neoplasms. Egypt J Otolaryngol 2015;31:98-104. DOI: 10.4103/1012-5574.156091.

3. Zafar U, Khan N, Afroz N, et al. Clinicopathological study of nonneoplastic lesions of nasal cavity and paranasal sinuses. Indian J Pathol Microbiol 2008;51(1):26-29. DOI: 10.4103/0377-4929.40386.

4. Bakari A, Afolabi OA, Adoga AA, et al. Clinico-pathological profile of sinonasal masses: an experience in national ear care center Kaduna, Nigeria. BMC Research Notes 2010;3:186. DOI: 10.1186/1756-0500-3186.

5. Lathi A, Syed MMA, Kalakoti P, et al. Clinico-pathological profile of sinonasal masses: a study from a tertiary care hospital of India. Acta Otorhinolaryngol Ital 2011;31(6):372-377.

6. Bist SS, Varshney S, Baunthiyal V, et al. Clinico-pathological profile of sinonasal masses: an experience in tertiary care hospital of
Uttarakhand. Natl J Maxillofac Surg 2012;3(2):180-186. DOI: 10.4103/0975-5950.111375.

7. Khan N, Zafar U, Afroz N, et al. Masses of nasal cavity, paranasal sinuses and nasopharynx: a clinicopathological study. Indian J Otolaryngol Head Neck Surg 2006;58(3):259-263.

8. Dasgupta A, Ghosh RN, Mukherjee C. Nasal polyps - Histopathologic spectrum. Indian J Otolaryngol Head Neck Surg 1997;49(1):32-36.

9. Tondon PL, Gulati J, Mehta N. Histological study of polypoidal lesions in the nasal cavity. Indian J Otolaryngol Head Neck Surg 1971;3:3-11.

10. Drake-Lee AB. Nasal polyps. Hospital Med 2004;65(5):264-267. DOI: 10.12968/hosp.2004.65.5.13699.

11. Bellanti J, Azem M, MacDowell-Carneiro A, et al. Possible mechanisms of late-life-onset allergic diseases and asthma in the senior citizen. Allergy Asthma Proc 2000;21(5):267-270. DOI: 10.2500/108854100778248287.

12. Bateman ND, Fahy C, Woolford TJ. Nasal polyps: still more questions than answers. J Laryngol Otol 2003;117(1):1-9. DOI: 10.1258/002221503321046577.

13. Chaaban MR, Walsh EM, Woodworth BA. Epidemiology and differential diagnosis of nasal polyps. Am J Rhinol Allergy 2013;27(6):473-478. DOI: 10.2500/ajra.2013.27.3981.

14. Bachert C, Gevaert P, Holtappels $G$, et al. Total and specific IgE in nasal polypsis related to local eosinophilic inflammation. J Allergy Clin Immunol 2001;107(4):607-614. DOI: 10.1067/mai.2001.112374.

15. Tan CS, Yong VK, Yip LW, et al. An unusual presentation of a giant frontal sinus Mucocele manifesting with a subcutaneous forehead mass. Ann Acad Med Singapore 2005;34(5):397-398.

16. Suri A, Mahapatra AK, Gaikwad S, et al. Giant mucoceles of the frontal sinus: a series and review. J Clin Neurosci 2004;11(2):214-218. DOI: 10.1016/j.jocn.2003.02.011.

17. Edelman RR, Hesselink JR, Zlatkin MB, et al. Clinical Magnetic Resonance Imaging. 3rd ed., Philadelphia: Elsevier; 2006. p. 2035.

18. Muzyka MM, Gubina KM. Problems of the epidemiology of scleroma. I. geographical distribution of scleroma. J Hyg Epidemiol Microbiol Immunol 1971;15:233-242.

19. Pradhananga RB, Adhikari $P$, Thapa NM, et al. Overview of nasal masses. J Inst Med 2008;30:13-16.

20. Zhong $Q$, Guo W, Chen $X$, et al. Rhinoscleroma: a retrospective study of pathologic and clinical features. J Otolaryngol Head Neck Surg 2011;40:167-174.

21. Hyams VJ. Papillomas of the nasal cavity and the paranasal sinuses, a clinicopathologic study of 315 cases. Ann Otol Rhinol Laryngol 1971;80(2):192-196. DOI: 10.1177/000348947108000205.

22. Lawson W, Biller HF. Treatment outcomes in the management of inverted papilloma: an analysis of 160 cases. Laryngoscope 2003;113(9):1548-1556. DOI: 10.1097/00005537-200309000-00026.

23. Vrabec DP. The inverted Schneiderian papilloma: a clinical and pathological study. Laryngoscope 1975;85(1):186-220. DOI: 10.1288/00005537-197501000-00014.

24. Banhiran W, Casiano RR. Endoscopic sinus surgery for benign and malignant nasal and sinus neoplasm. Curr Opin Otolaryngol Head Neck Surg 2005;2(2):113-117. DOI: 10.1097/00020840-20050200000012.

25. Synder RN, Perzin KH. Papillomatosis of nasal cavity and paranasal sinuses (inverted papilloma, squamous papilloma): a clinicopathologic study. Cancer 1972;30(3):668690. DOI: 10.1002/1097-0142(197209)30:3<668::AIDCNCR2820300315>3.0.CO;2-B.

26. Saha SN, Ghosh A, Sen S, et al. Inverted papilloma: a Clinicopathological dilemma with special Reference to Recurrence and malignant transformation. Indian J Otolaryngol Head Neck Surg 2010;62(4):354-359. DOI: 10.1007/s12070-010-0056-y. 